\title{
IEEE body area networks and medical implant communications
}

\section{Bin Zhen}

National Institute of Information and Communications Technology

3-4 Hikarino-oka, Yokosuka, Japan zhen.bin@nict.go.jp
Huan-Bang Li

National Institute of Information and Communications Technology

3-4 Hikarino-oka, Yokosuka, Japan lee@nict.go.jp
Ryuji Kohno

Yokohama National University

79-5 Tokiwadai, Yokohama, Japan kohno@ynu.ac.jp

\begin{abstract}
IEEE 802.15 established a new working group, wireless body area networks (WBAN), to develop short range wireless technology in and around human body recently. This paper investigates networking issues in implant communications of WBAN. The object is better understanding of medical implant sensor networks and how to start WBAN's work. We applied IEEE 802.15.4b and 802.15.4a-chirp spread spectrum (CSS) for implant communications. We found two issues: clear channel assessment of implant devices and adjacent-channel interference from free space signals. Both of them can be attributed to the rapid attenuation of electromagnetic wave through tissues. Therefore the carrier sense multiple access mechanism and transmit mask of 802.15.4b cannot be directly adopted. The modulation of $802.15 .4 a-C S S$ is a good reference to WBAN. Besides, a simple two-hop protocol which uses a body surface forwarder was presented for long distance wireless implant communications.
\end{abstract}

\section{Categories and Subject Descriptors}

C.4 [Performance of Systems] - Reliability, availability, and serviceability.

\section{General Terms}

Standardization, Performance.

\section{Keywords}

Body area networks, carrier sense, medium access control

\section{INTRODUCTION}

Medical implants have a history of outstanding success in the monitor of patient's condition and in the diagnosis and treatment of many diseases, including heart disease, gastrointestinal tract, neurological disorders, cancer detection, handicap rehabilitation and general health monitor. The wireless link between external device and medical implant enables a doctor to reprogram therapy and obtain useful diagnosis information; the link among implant devices enables in vivo timely reactive treatment.

Recently IEEE 802.15 working group established a new task group, wireless body area networks (TG-WBAN), which will to develop guideline for using short range wireless technologies in various healthcare and consumer services [1]. The main advantages of wireless system over wired alternative include enhanced physical mobility, reduced risk of infection and failure, less invasion and lower cost of care delivery. Other considerations include easy and cost-reduced deploying and interoperability between networked devices. The WBAN considers both wearable sensors on the surface of body and medical implant surgically placed inside of patient's body. To enter the next step of standardization, TGWBAN must decide whether to define a new PHY/MAC or an enhancement of IEEE 802 wireless personal area networks (WPAN) standards. A new standard from scratch to satisfy all needs of healthcare may be not an economical option. Because none of IEEE 802 standard is intended or designed for medical and implantable communication, it is unknown how efficient the WPAN technologies can support the life critical wearable and implantable medical applications. TG-WBAN therefore needs to evaluate current available or emerging IEEE 802 low data rate wireless technologies. There are a number of fundamental questions: What are the requirements from WBAN applications? Can IEEE 802 wireless technologies fulfill medical and healthcare requirements? In which cares/environment do they not work? Where are the performance bottlenecks?

This paper introduces IEEE WBAN and investigates networking issues in implant communications. The reminder of paper is organized as follows. Section II describes IEEE WBAN and medical implant applications. Section III gives a brief review of wireless implant communications. In section IV we analyze the networking issues of implant communication in a piconet and among piconets. Section V finally concludes the paper.

\section{IEEE WBAN}

\section{A. IEEE WBAN}

Historically BAN was first discussed under the topic of PAN. Zimmermann is credited with inventing the concept of BAN based on his work at MIT and later at IBM [2]. He discussed a combination of portable computing devices and short range wireless link as providing a new paradigm for computing and communication. The link can be established through handshake and communication was made by direct touch by close vicinity $(<2 \mathrm{~m})$. In the first version of WWRF Book of Vision, PAN was shown as the innermost sphere near to the user [3]. In 2004, BAN was defined as immediate environment around people which includes those 'nearest' object that might be part of body.

IEEE WBAN was not created in a vacuum. It is a natural extension of IEEE 802 standards from metropolitan area network (MAN), to LAN and to PAN. WBAN will address a unique solution for body area networks that provide short-range communications in and around human body with consideration for human body safety. It targets a convergence of sensors/actuators and wireless communication in healthcare and consumer devices.

Although WBAN and WPAN are close relatives, they are different in the definition of IEEE WBAN [1]. Because WBAN device can be physically on the surface or inside of a person's body, the safety to human/animal body is therefore the first factor taken into considered. Both wearable and implantable WBAN devices must be conscious of specific absorption rate (SAR) to protect human tissue. On the other hand, WPAN devices are only close to the user. Wearable IEEE WPAN devices are suggested to be separated at least $30 \mathrm{~cm}$ distance from human body. WBAN channel is different from that of WPAN. The body surface channels mainly depend on space wave and surface wave propagation [4]. A new floor reflection component and fluctuation in received energy due to body motions were observed. The implant communication is not considered in WPAN. As tissue medium of humans is lossy and mainly consists of salt water, the propagation of electromagnetic wave attenuates much faster than that in free space [5]. In addition the fading is frequency dependent and is strongly influenced by the layered body structure. And since WBAN devices are physically on the surface or inside of a body, they are in the near field of an antenna. The antenna pattern can be affected by new border conditions, e.g. tissue can absorb part of radiated radio energy. It has observed that antenna height has a major influence on the path loss in body surface channel [6]. Furthermore, medical signals require guaranteed latency and accuracy to external stimuli since some of them are life critical.

IEEE 1073 committee is currently developing a complete seven layers "medical information bus" for wireless data communication among point-of-care medical devices $[7,8]$. The main objective is to define universal and interoperable interface that are transparen to end user, easy to use and self-configurable. This is different from IEEE WBAN which only focuses on PHY and MAC interface. Figure 1 maps the specifications defined IEEE WBAN and IEEE 1073 in the ISO layer model. Besides, IEEE 1073 group is not so much keen on developing new wireless technologies.

\begin{tabular}{|c|c|c|}
\hline ISO model & EE 1073 & \\
\hline Application & \begin{tabular}{|l} 
Medical device data \\
language
\end{tabular} & P1073.1 \\
\hline Presentation & & \\
\hline Session & $\begin{array}{l}\text { Device appilcation } \\
\text { profile }\end{array}$ & P1073.2.x \\
\hline Transport & & \\
\hline Network & Transport profile & P1073.3.x \\
\hline Data link & \begin{tabular}{|l|l|}
$\mathrm{IEE}$ \\
\end{tabular} & \\
\hline Physical & & P1073.4.x \\
\hline
\end{tabular}

Fig. 1 Topology architecture of IEEE 1073

\section{B. Wireless medical sensor networks}

The medical and healthcare applications distinguish WBAN from other working groups in IEEE 802 committee. Table I compares medical WBAN sensor networks with general wireless sensor networks (WSN) which are mainly implemented by low-rate WPAN technologies. The common features include limited resources (e.g. computation power, memory, battery, bandwidth), low/modest duty cycle, energy efficiency, plug-and-play, diverse coexistence environments, and heterogeneous device ability. We also found significant differences in sensor device, dependability, 
networking, traffic pattern and channel.

Firstly medical sensors consider safety, quality and reliability as top priority, while general WSN are cost sensitive for marke reason. To improve reliability, general WSN tend to distribute redundant sensors as backup for sensing, transmission and forwarding. In contrast, there is little redundancy in medical WSN for medical reasons. Vital signals, like ECG (Electrocardiogram) and EEG (Electroencephalography) are location dependent and can only be measured by deterministic location. Thus it is difficult to allocate redundant sensors in the limited area. Especially, it makes no sense to allocate sensors outside of the interest/effect area.

Secondly the medical sensor networks have more frequency bands to select than general WSN, which usually work in IndustryScience-Medical (ISM) band. Although the specific medical bands are less noisy, they are narrow band and conditional license. For example, the wireless medical telemetry service (WMTS) band can only be used in the licensed hospital and clinic, but not at home.

Thirdly, the traffic pattern in medical sensor networks is featured by periodical real time data (e.g. EEG and ECG) and some top priority burst data (e.g. alarm and alert) [9]. In contrast, general WSN typically consider versatile traffic. The medical information, especially the alarm notification, have very strict requirement in terms of QoS, which are usually more stringent QoS than general WSN. It is well know that the distributed carrier sense multiple access (CSMA) mechanism cannot provide guaranteed QoS.

The lack of redundancy, priority traffic, dominant periodical data and guaranteed QoS in versatile coexistence environment challenge the reliability design of PHY and MAC of IEEE WBAN.

\section{Medical implants of WBAN}

The wireless interface of medical implant is challenged by its unique and fundamental difference from other WSN applications domain [10]. Medical implants may have more stringent limitation in size and weight, and therefore limited processing, memory and power capacities. However, lifetime of implant devices which are usually in continuous operation must be maximized to avoid the risk, cost and patient trauma inherent in replacement surgical procedure. Power management of low power transceiver, processor and sensor/actuator, and sometimes energy harvest are necessary. Furthermore, the material used should be biocompatibility with human body since human immune system will combat foreign substances in the body. Location of implant is another challenge. A medical implant will be located by physician to where it provides the best patient care and comfort, with little consideration on the radio propagation and network. Figure 2 depicts WBAN implants and the implementation concerns [11].

Because of the strong heterogeneity of implant devices, the data rate of implant communication varies strongly, ranging from simple data of a few kbps in pacemaker to several Mbps in capsular endoscope. Usually communication is between implant devices and external controller (base station). The dominant data stream is from implant device to external controller or vice versa, e.g. camera capsule and neuro-stimulator. In a closed-control application, e.g., a glucose sensor and insulin pump for diabetes, communications occur among implant devices.

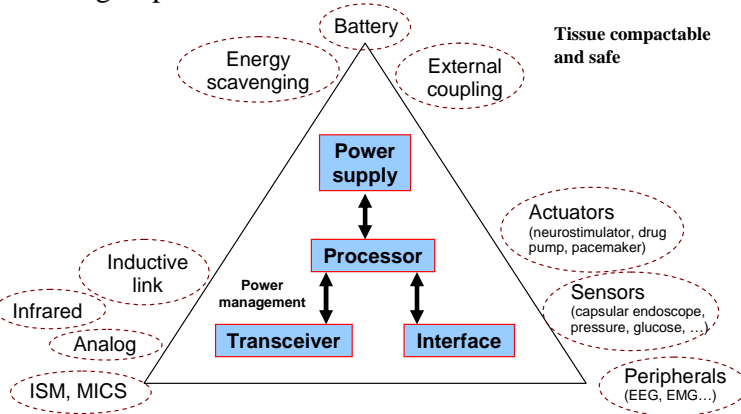

Fig. 2 WBAN implant and concerns of implementation

\section{WIRELESS MEDICAL IMPLANT COMMUNICATION}

As stated in last section, one of the key issues of wireless implant is power consumption. This has been studied in component level, device level, network level, and application level. Federa Communication Committee allocated a frequency band in 402 $405 \mathrm{MHz}$ for medical implant communication service (MICS) on a shared, secondary basis in 1999. This frequency band best meets the technical requirements of implant communications for a number of reasons. Before that, medical implants depended on magnetic coupling in the low frequency (LF) band, which require that the implanted device be in very close vicinity of outside controller. Other frequencies considered for implant communications include $916 \mathrm{MHz}, 1.5 \mathrm{GHz}$ and Ultra-Wideband [12-14]. Gupta et al asserted that classic open-air radio models are not applicable to implant network [5]. The proposed propagation model considered antenna, media and power loss due to tissue absorption. Tang et al presented a minimum energy coding based On-Off Keying with coherent receiver for retina prosthesis [15]. Timmons and Scanlon showed IEEE 802.15.4 can be used for medical sensor networks when properly configured [16]. Tang et al even considered thermal effects in the routing protocol of mesh biosensor networks [17]. Hybrid of chain and cluster based network architecture is more efficient than tree-based approach [18].

Compared with the state of art MICS defined systems, an 802.15.4b and $802.15 .4 \mathrm{a}$ based WBAN in $2.4 \mathrm{GHz}$ ISM band would go beyond for peer-to-peer networking support, wide bandwidth and mature chip design. Each IEEE 802.15.4b channels has $5 \mathrm{MHz}$ bandwidth to provide $250 \mathrm{kps}$ data rate [20]. The basic MAC mechanism is CSMA. The medium idleness is evaluated during a CCA (Clear Channel Assessment) period of time. CCA can be a detection of energy above a threshold or modulation and spreading characteristic detection. The CSS (chirp spread spectrum) PHY of IEEE 802.15.4a provides enhanced immunity to multipath fading and extended range with very low transmit power [21]. A chirp is a linear frequency modulated pulse which sweeps the band at a very high speed. Its channel plan is identical to that of $802.11 \mathrm{~b}$ systems. The default data rate is up to $1 \mathrm{Mbps}$. Because of its frequency sweeping nature, 802.15.4a-CSS system adopts ALOHA for channel access.

\section{NETWORKING ISSUES IN WIRELESS IMPLANT COMMUNICATIONS}

\section{A. Analysis scenarios and assumptions}

Figure 3 depicts the analysis scenarios adopted in this paper. Each person establishes its own piconet in a star topology, where an externally worn controller acts as coordinator to gather vital signals and forward them to infrastructure network via other links. The implanted medical sensors are devices of the piconet. We considered two cases: a single piconet and multiple piconets in a close space, for example, several patients live in a big medical ward or stay in a clinic.

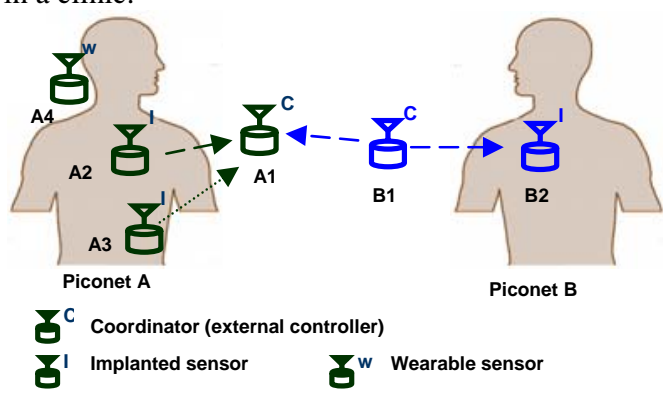

Fig. 3 Analysis scenario of implant communications

Transmission powers of all devices are $0 \mathrm{dBm}$. We assumed that all implants are 20mm under skin surface. The corresponding tissue attenuation plus antenna matching was assumed to be $-35 \mathrm{~dB}$ [5]. ${ }^{1}$ The free-space path loss measured in $\mathrm{dB}$ is

$$
p l=40.2+20 \log _{10}(d),
$$

where the distance $d$ is smaller than 8 meters. The total path loss from implant to external controller is the sum of tissue loss and free space loss. The bit error rate (BER) of $802.15 .4 \mathrm{~b}$ in additive white Gaussian noise (AWGN) environment is

$$
B E R=\frac{8}{15} \times \frac{1}{16} \times \sum_{k=2}^{16}-1^{k}\left(\begin{array}{l}
16 \\
k
\end{array}\right) e^{20 S N R(1 / k-1))},
$$

where SNR is the signal-to-noise ratio. For 802.15.4a-CSS running at $1 \mathrm{Mbps}$, the BER becomes

$$
B E R=\frac{6 \times Q\left(\sqrt{3 \times S N R_{0}}+Q\left(\sqrt{6 \times S N R_{0}}\right)\right)}{2},
$$

where $S N R_{0}=S N R \times 14 \times 1.667$. We did not consider any other noise source except packet collision in channel access.

\section{B. Networking issues of IEEE 802.15.4b}

Figure 4 describes the reception power at the external coordinator. The horizon axis is the distance from body surface to coordinator in free space. Considering a $-85 \mathrm{dBm}$ receiver sensitivity, the maximal

Tissue attenuation increases with distance and frequency. More power is absorbed in the near-field of antenna and in the case of a tissue with more water content [5]. 
distance is about 3 meters. But there is no link budget for some worse cases, e.g. an implant may be behind a bone.

We first considered CCA ability. As shown in Fig. 3, the channel is occupied by an implant sensor A2. The free space sensors A4 and $\mathrm{B} 1$, and another implant A3 detect the channel state through CCA operation. Because there is no specified CCA sensitivity in the standards, we assume two CCA thresholds: $-85 \mathrm{dBm}$ and $-95 \mathrm{dBm}$. A channel is considered to be free if radio signal strength is below the threshold. The circle-line in Fig. 5 draws the CCA ability of A4. Wearable sensors cannot 'see' the activity of implant when it is over 3 meters away from body surface given a $-85 \mathrm{dBm}$ CCA threshold. The 3 meters distance can guarantee the correct CCA sensing of activity of implants in the same piconet (We do not consider the shadowing of channel induced by body movement.) But in the multiple piconets case, the distances between implants and wearable sensors in another piconet, e.g. node $\mathrm{B} 1$ in piconet $\mathrm{B}$ can be more than 3 meters sometimes. Another $-95 \mathrm{dBm}$ threshold presents a much better CCA performance. The CCA range of wearable sensors is about 9 meters. The square-line in Fig. 5 shows the CCA ability of implant device A3 in the same piconet. We assumed that the radio signal propagates from implant to body surface and enters body again. ${ }^{2}$ The free space is only 0.5 meter even given a $-95 \mathrm{dBm}$ CCA threshold. The distance is not enough even in a piconet. Usually the radio propagation and network are not considered by physician who put a medical implant into patients. For example, in the diabetes treatment, an implanted glucose sensor is buried under skin in the arm to measure blood sugar level, while the implanted insulin pump is put in the abdomen. It is unpractical to limit the distance between medical implants within $0.5 \mathrm{~m}$ in real applications.

Therefore, although the wearable device's CCA of at body surface works well, the implant device's CCA is not reliable. This is because path losses in tissue are much bigger than those in free space. All implants which have failed CCA become "hidden nodes" to the transmitter, which contend channel with transmitter in an ALOHA way, which is known for its low throughput and power inefficient. This means CSMA does not work well in implantable WBAN. However, CCA of implanted devices is assumed to be reliable in [16]. Although research on "hidden nodes" is a hot topic in wireless ad hoc network, it is unknown the power consumption of the proposed methods. An alternative solution is to adopt a time division multiple access approach instead as suggested in [9].

We then considered implant communication in the multiple piconets environment. For example, as shown in Fig. 3, when node A2 is transmitting to node A1, node B1 may communicate with node B2 at the same time. Packets from A2 and B1 may collide at node A1 when CCA fails. We specified a 1 meter distance between A1 and A2. Figure 6 plots the BERs received at A1 when packet collision occurs (We did not consider background noise). The horizontal axis is the separation distance between A1 and B1. In the case two piconets works in the same channel, as shown in the blue line, it is almost impossible for a smooth communication. ${ }^{3}$ The BER is more than $1 \%$ even when B1 is 20 meters away from A1. The square-line in Fig. 5 describes a case where two piconets operate in adjacent channels, where the transmit mask is $20 \mathrm{~dB}$ attenuation outside channel as required in the standard. To achieve $0.1 \% \mathrm{BER}$, the separation distance should be larger than 5 meters. In real implementation, typical transmit mask is more than $20 \mathrm{~dB}$. Given a $25 \mathrm{~dB}$ relative power attenuation, as indicated by the starline, the separation distance should still be larger than 3 meters This separation between WBAN piconets seems hard to be guaranteed in some environments, e.g. clinic and ward. During network formation phase of $802.15 .4 \mathrm{~b}$, the coordinator scans all channels to find an unoccupied one to work in. There is no requirement that two piconets cannot work in adjacent channels. As shown in Fig. 6(a), the free space signals in adjacent channel can severely interfere to the implant communications. This is because the tissue attenuation may be bigger than the spectrum mask. Therefore WBAN requires a more stringent out-band attenuation than that defined by $802.15 .4 \mathrm{~b}$.

\section{Networking issues of IEEE 802.15.4a-CSS}

Because of the same working frequency, the reception power at external coordinator of 802.15.4a-CSS can also be described by Fig. 4, which is sufficient to reach beyond 3 meters and can provide enough link budget. Figure 6(b) describes the BER of packe collision between two piconets. Different from 802.15.4b, the free

${ }^{2}$ As shown in Fig. 7 of [5], the path loss between two implants through tissue is more than $90 \mathrm{~dB}$ when distance is larger than $110 \mathrm{~mm}$. A direct through body path between implants usually is not available.

${ }^{3} \mathrm{~A}$ smooth communication means the link BER is less than $0.1 \%$. space signals in adjacent channel with $25 \mathrm{~dB}$ attenuation cannot severely interfere to the implant signals. The chirp modulation is robust. There is no CCA issue in 802.15.4a-CSS system because of the ALOHA channel access. However, an ALOHA system suffers from in-efficiency when the piconet is heavily loaded.

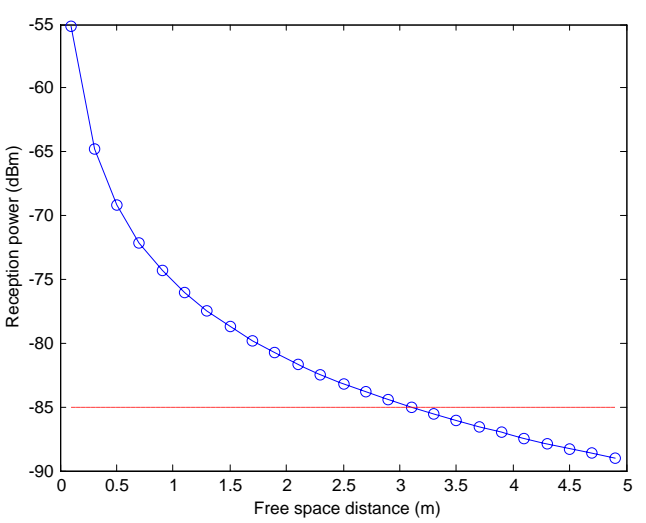

Fig. 4 Received power level at external coordinator

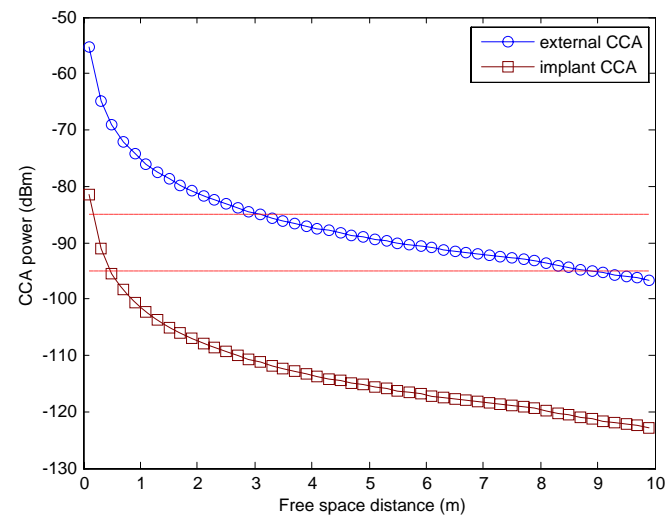

Fig. 5 CCA of medical implant devices
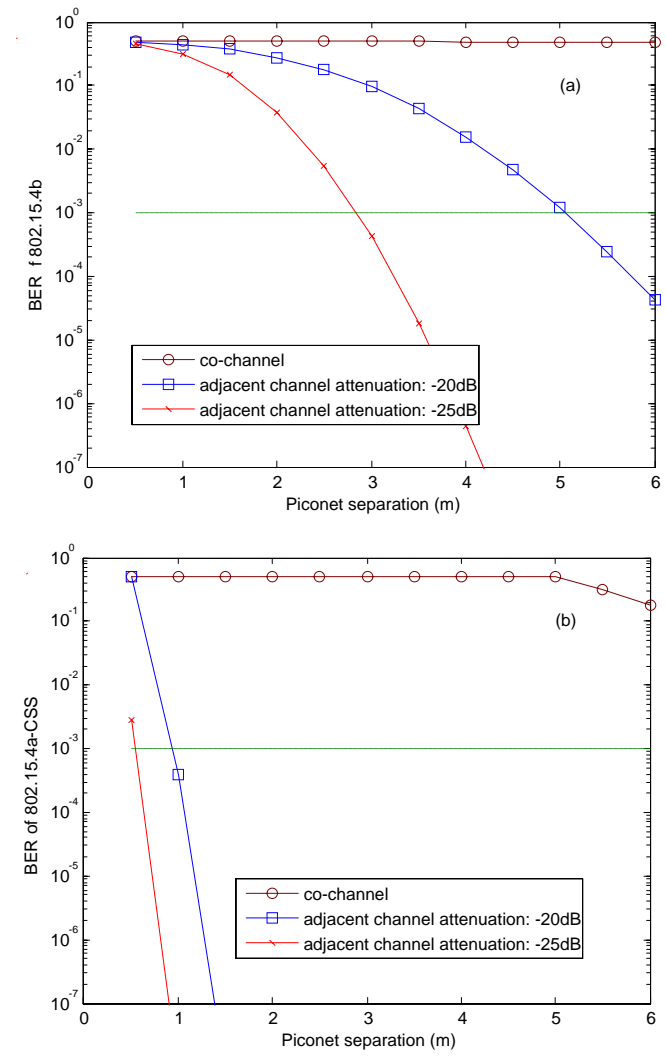

Fig. 6 BER received at A1 in the coexistence of BAN piconets: 802.15.4b PHY (a), and 802.15.4a-CSS PHY (b)

\section{Long distance implant communications}

Long distance communication among implant devices was considered in [10, 17-19]. The motivation is to balance thermal effect resulting from communication radiation and power dissipation by routing data through multihop. The basic idea is to organize implant sensors into cluster and route data using SAR minimization metrics. Most of them are extension of energy efficient routing in general WSN.

In the implanted scenario considered in WBAN, as shown in Fig. 3 , there is always an externally worn coordinator which is outside of body. On considering the significant difference of radio attenuation in tissue and free space, it is intuitive to select body surface 
coordinator as a message forwarder. Data from implant sensor first goes to the out-of-body forwarder and is then forwarded back into body to the destination. Several benefits can be immediately found. A body surface device has less limitation than implant devices. Even battery recharge is practical possible. This gives more freedom in channel access, routing and management design. The body surface coordinator is natural manager of total network. Communication between any pair of implanted devices can be researched within two hops. No complex routing algorithm and distributed network management are needed. And the thermal effect is simple and constant.

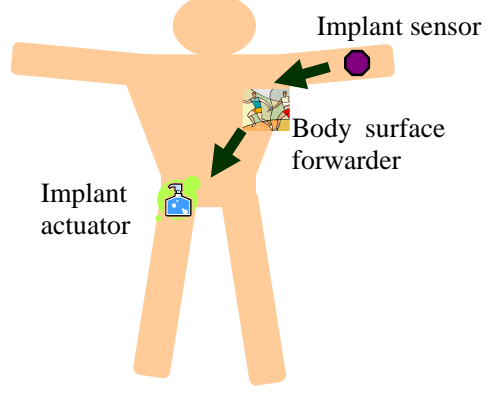

Fig. 7 Two-hop protocol for long distance wireless implants communications

\section{CONCLUSION}

In conclusion, we introduced IEEE WBAN and explored IEEE WPAN technologies, for medical implant communications. It is an initial effort of WBAN's work. The CCA analysis showed that the CCA range of implant is only 0.5 meter. The unreliable CCA of implant indicates that CSMA cannot be adopted in the implant WBAN. Another analysis revealed that the free space signals in the adjacent channel may also threaten the implant communications. Implant communication requires more stringent spectrum mask and robust modulation than that of $802.15 .4 \mathrm{~b}$. Both of them can be attributed to the rapid attenuation of electromagnetic wave through the lossy tissues. From this we concluded that $802.15 .4 \mathrm{~b}$ is not a good reference for WBAN. In contrary, the 802.15.4a-CSS PHY is more suitable to be considered. We presented a simple two-hop protocol, which use a body surface forwarder, for long distance communications between implants.

\section{REFERENCES}

[1] A.W. Astrin, "SG-BAN project authorization request draft," IEEE 15-06-0408-00-0ban, (work in progress).

[2] T.G. Zimmerman, "Personal area network: near-field intrabody communication,” IBM Systems J., vol.38, no.4, p.566-574, 1999.

[3] Wireless World Research Forum, "The book of vision 2001: vision of the wireless world 2001".

[4] A. Fort, C. Desset and P. De Doncker et al, "An Ultrawideband body area propagation channel model- from statistics to implementation," IEEE Tran. Microwave Theory and Techniques, vol.54, no.4, p.1820-1826, 2006.

[5] S.K.S. Gupta, S. Lalwani and Y. prakash et al, "Towards a propagation model for wireless biomedical applications," IEEE Inter. Conf. Communications, vol.3, p.1993-1997, 2003.
[6] L. Roelence, S.V.D. Bulcke, and W. Joseph, et al, "Path loss model for wireless narrowband communication above flat phantom,” Electronics Letters, vol.42, no.5, p.10-11, 2006.

[7] R. Schrenker and T. Cooper, "'Building the Foundation for Medical Device Plug-and-Play Interoperability"," Medical Electronics Manufacturing, 2001.

[8] IEEE P1073.0.1.1/D01J, "Draft guide for health informaticsPoint-of-care medical device communication-technical reportGuidelines for the use of RF wireless technology,” 2006.

[9] B. Zhen, M. Patel, S. Lee and E. Won, "Body area networks (BAN) technical requirements," IEEE 802.15.BAN proposal (07-0867-03), 2007.

[10] L. Schwiebert, S.K.S. Gupta, and J. Weinmann, "Research challenges in wireless networks of biomedical sensors," ACM Conf. on Mobile Computing and Networking, p.151-165, 2001.

[11] K.A. Townsend, J. W. Haslett, and T.K.K. Tsang et al, "Recent advances and future trends in low power wireless systems for medical application," $5^{\text {th }}$ Inter. Workshop on System-on-Chip for Real-time Applications, p.476-481, 2005.

[12] W.G. Scanlon, J.B. Buirns, and N.E. Evans, "Radiowave propagation from a tissue-implanted source at $418 \mathrm{MHz}$ and 916.5MHz,” IEEE Trans. on Biomedical Engineering, vol.47, no.4, p.527-534, 2000.

[13] J. Wang and D. Su, "Design of an Ultra-wideband system for in-body wireless communications," $4^{\text {th }}$ Asia-Pacific Conf. on Environmental Electromagnetic, p.565-568, 2006.

[14] J. Kim and Y. Rahmat-Samii, "Electromagnetic interactions between biological tissues and implantable biotelemetry systems," IEEE Inter. Microwave Symposium, vol.47, no.4, p.1801-1804, 2005.

[15] Q. Tang. S.K.S. Gupta, and L. Schwiebert, "BER performance analysis of an On-Off Keying based minimum energy coding for energy constrained wireless sensor application," IEEE Inter. Conf. Communications, vol.4, p.2734-2738, 2005.

[16] N.F. Timmons and W.G, Scanlon "Analysis of the performance of IEEE 802.15.4 for medical sensor body area networking," IEEE Conf. on Sensor and Ad Hoc Communications and Networks, p.16-24, 2004.

[17] Q. Tang, N. Tummala, and S.K.S. Gupta et al, "Communication scheduling to minimize thermal effects of implanted biosensor networks in homogeneous tissue," IEEE Trans. on Biomedical Engineering, vol.52, no.7, p.1285-1294, 2005.

[18] M. Moh, B.J. Culpepper, and L. Dung, et al, "On data gathering protocols for in-body biomedical sensor networks," IEEE Global Communications Conf., vol.5, p.2991-2996, 2005.

[19] Q. Tang, N. Tummala, and S.K.S. Gupta et al, “TARAThermal-aware routing algorithm for implanted sensor networks," Lecture Notes in Computer Science, vol.3560, p.206-217, 2005.

[20] IEEE standard 802.15.4b-2006.

[21] IEEE standard 802.15.4a-2007.

Table I Comparison between medical sensor networks and general wireless sensor networks

\begin{tabular}{|c|c|c|}
\hline & WBAN medical sensor networks & General wireless sensor networks \\
\hline $\begin{array}{l}\text { Common } \\
\text { features }\end{array}$ & \multicolumn{2}{|c|}{$\begin{array}{l}\text { Limited resources: battery, computation, memory, energy efficiency } \\
\text { Diversity coexistence environment } \\
\text { low/modest data rate, low/modest duty cycle } \\
\text { Dynamic network scale, plug-and-play, heterogeneous devices ability, dense distribution }\end{array}$} \\
\hline $\begin{array}{l}\text { Sensor/ } \\
\text { actuator }\end{array}$ & $\begin{array}{l}\text { Single-function device } \\
\text { Fast relative movement in small range } \\
\text { device lifetime, } \\
\text { days, }<10 \text { years (implant sensor) } \\
\text { Safe (low SAR) and quality first } \\
\end{array}$ & $\begin{array}{l}\text { Multi-function device } \\
\text { Rare or slow movement in large range } \\
\text { network lifetime and device lifetime, } \\
\text { months, }<10 \text { years } \\
\text { Cost sensitive }\end{array}$ \\
\hline Dependability & $\begin{array}{l}\text { Reliability (first), guaranteed QoS } \\
\text { Strongly security (except emergency) }\end{array}$ & $\begin{array}{l}\text { expected QoS, redundancy-based reliability } \\
\text { Required security }\end{array}$ \\
\hline Networking & $\begin{array}{l}\text { Small scale star network } \\
\text { No redundancy in device } \\
\text { Deterministic node distribution }\end{array}$ & $\begin{array}{l}\text { Large scale hierarchical network } \\
\text { redundant distribution } \\
\text { Random node distribution }\end{array}$ \\
\hline Traffic & $\begin{array}{l}\text { Periodical RT (dominant), burst (priority) } \\
\text { Uni-directional traffic } \\
\text { M:1 communication }\end{array}$ & $\begin{array}{l}\text { Burst (dominant), periodical } \\
\text { Uni-directional or bi-directional traffic } \\
\text { M:1 or point-point communication }\end{array}$ \\
\hline channel & $\begin{array}{l}\text { Specific medical channel, ISM band } \\
\text { Body surface or through body }\end{array}$ & $\begin{array}{l}\text { ISM band } \\
\text { Obstacle is unknown }\end{array}$ \\
\hline
\end{tabular}

